\title{
The spectroscopic evolution of the symbiotic star AG Draconis
}

\section{The $O$ VI Raman, Balmer, and helium emission line variations during the outburst of 2006-2008}

\author{
S. N. Shore ${ }^{1,2}$, G. M. Wahlgren ${ }^{3,4}$, K. Genovali ${ }^{1}$, S. Bernabei ${ }^{5}$, P. Koubsky ${ }^{6}$, M. Šlechta ${ }^{6}$, P. Škoda ${ }^{6}$, \\ A. Skopal ${ }^{7}$, and M. Wolf ${ }^{8}$ \\ 1 Dipartimento di Fisica "Enrico Fermi”, Università di Pisa, largo B. Pontecorvo 3, 56127 Pisa, Italy \\ e-mail: shore@df.unipi.it \\ 2 INFN - Sezione di Pisa, Italy \\ 3 Catholic University of America, Dept. of Physics, 620 Michigan Ave NE, Washington DC, 20064, USA \\ 4 NASA-GSFC, Code 667, Greenbelt, MD, 20771, USA \\ 5 INAF - Osservatorio Astronomico di Bologna, via Ranzani 1, 40127 Bologna, Italy \\ 6 Astronomical Institute, Academy of Sciences of the Czech Republic, Ondřejov, Czech Republic \\ 7 Astronomical Institute, Slovak Academy of Sciences, 05960 Tatranskńa Lomnica, Slovakia \\ 8 Astronomical Institute, Faculty of Mathematics and Physics, Charles University Prague, 18000 Praha 8, V Holešovičkách 2 , \\ Czech Republic
}

Received 29 September 2009 / Accepted 23 November 2009

\section{ABSTRACT}

\begin{abstract}
Context. AG Dra is one of a small group of low metallicity S-type symbiotic binaries with K-type giants that undergoes occasional short-term outbursts of unknown origin.

Aims. Our aim is to study the behavior of the white dwarf during an outburst using the optical Raman lines and other emission features in the red giant wind. The goal is to determine changes in the envelope and the wind of the gainer in this system during a major outburst event and to study the coupling between the UV and optical during a major outburst.

Methods. Using medium and high resolution groundbased optical spectra and comparisons with archival FUSE and HST/STIS spectra, we study the evolution of the Raman O VI features and the Balmer, He I, and He II lines during the outburst from 2006 Sep. through 2007 May and include more recent observations (2009) to study the subsequent evolution of the source.

Results. The O VI Raman features disappeared completely at the peak of the major outburst and the subsequent variation differs substantially from that reported during the previous decade. The He I and He II lines, and the Balmer lines, vary in phase with the Raman features but there is a double-valuedness to the He I 6678, 7065 relative to the O VI Raman $6825 \AA$ variations in the period between 2006-2008 that has not been previously reported.

Conclusions. The variations in the Raman feature ratio through the outburst interval are consistent with the disappearance of the O VI FUV resonance wind lines from the white dwarf and of the surrounding $\mathrm{O}^{+5}$ ionized region within the red giant wind provoked by the expansion and cooling of the white dwarf photosphere.
\end{abstract}

Key words. stars: individual: AG Draconis - binaries: symbiotic - radiation mechanisms: general - atomic processes

\section{Introduction}

Although as a class, the symbiotic stars are enigmatic, the S-type star AG Draconis is unusually full of surprises. The system has an orbital period of approximately 550 days (Meinunger 1979; Fekel et al. 2000) and consists of a low metallicity K0K3 giant (see, e.g., Smith et al. 1996) and a hot white dwarf (WD), whose effective temperature is estimated to be between $100 \mathrm{kK}$ and $170 \mathrm{kK}$. Although there are no eclipses, the ultraviolet (UV) variations are consistent with orbital modulation due to wind absorption of the WD continuum and its associated ionized region by the giant (Gonzalez-Riestra et al. 1999, hereafter G99; Young et al. 2005). The archival photometric history of AG Dra is particularly interesting. For instance, ten major outbursts have been recorded for AG Dra, with a $V$ of 2 mag, between 1890 and 1960 (Robinson 1969) at intervals of roughly 14 to 15 years (Viotti et al. 1983; Mikolajewska et al. 1995; Tomov \& Tomova 2002; Leedjärv et al. 2004, hereafter L04;
Gális et al. 2004; Viotti et al. 2007; Skopal et al. 2007). The major events are double-peaked, with interburst intervals of $\approx 1 \mathrm{yr}$. Since the outburst of 1980 , additional bursts of weaker magnitude have been recorded that show a similar inter-outburst timescale. It is the strongest "supersoft" X-ray source known among the symbiotics (Greiner et al. 1997; Gonzalez-Riestra et al. 2008, hereafter G08) and is strongly variable with two characteristic states of hardness. Gonzalez-Riestra et al. (1999) distinguish between "cool" and "hot" outbursts, where the major outbursts of 1980 and 1994 were "cool" according to the He II Zanstra temperatures. These were interpreted as a cooling of the WD as its radius increases, leading to higher luminosity with envelope nuclear burning. Observations of AG Dra taken over the lifetime of $I U E$, and including the outburst episodes of 1980 and 1994, have been published by Gonzalez-Riestra et al. (1999). They note that the long wavelength UV continuum flux decreased by $20 \%$ over the observation baseline. Zanstra temperatures are in the range $90 \mathrm{kK}$ to $110 \mathrm{kK}$. Eriksson et al. (2006) 
determined WD wind velocities of 120 to $150 \mathrm{~km} \mathrm{~s}^{-1}$ based on a re-analysis of the $I U E$ high resolution spectra.

There is also a significant far ultraviolet flux (FUV) archive for AG Dra $(\lambda<1200 \AA)$, virtually all of it from quiescent intervals. ORFEUS spectra, obtained in 1993 with $B E F S$, show a broad O VI $1031 \AA$ line with peak flux of $3 \times$ $10^{-12} \mathrm{erg} \mathrm{s}^{-1} \mathrm{~cm}^{-2} \AA^{-1}$ along with strong narrow red giant wind components $\left(>10^{-11}\right)$. TUES spectra from 1996 showed a broad O VI line with peak flux $4 \times 10^{-12}$ (Schmid et al. 1999). HUT spectra obtained in 1995 show the broad line with peak flux $6 \times 10^{-12}$ but at comparatively low resolution (Birriel et al. 2000). FUS E spectra were taken during quiescent periods from March 2000 to December 2004 and in April 2007 during the decay of the 2006 outburst. In these data, Young et al. (2006) identified emission lines from highly ionized species, consistent with an electron temperature $T_{\mathrm{e}} \approx 20-30 \mathrm{kK}$. The high ionization Ne lines indicate a higher temperature closer to the WD. The broad O VI line was clearly variable even during periods with the same $U$ magnitude; the difference appears to be when the observations occurred relative to the outbursts and the type of outburst. Spectra were obtained with a range from $(<0.5$ to 5$) \times 10^{-12}$, the weak line occurred at the last epoch. Notably, BEFS and FUSE detected P Cyg components from the K star wind whenever the broad line was strong. One HST/STIS Ly $\alpha$ spectrum shows a broad, strong emission profile with $\mathrm{P}$ Cyg absorption at greater than $-2000 \mathrm{~km} \mathrm{~s}^{-1}$ at about the same time as the 2003 FUSE spectrum, a feature unobservable with $I U E$. The Lyman decrement is very large: no emission is ever detectable at Ly $\gamma$ and it is weak at $\mathrm{Ly} \beta$ in all FUV spectra.

The latest major outburst of AG Dra began in late August 2006 (around MJD 53900), earlier than anticipated from the average time between major outbursts, and reached a maximum of $V \approx 8$. This outburst was followed by a second, smaller one in October 2007 that showed a more rapid decline and reached a maximum $V \approx 8.8$. Unlike the previous two major outburst events, the UV spectrum (900 to $3000 \AA$ ) was not accessible with spectroscopy. However, the optical O VI $\lambda \lambda 6825,7080 \AA$ features are formed by Raman scattering on $\operatorname{Ly} \beta$ of the FUV $\mathrm{O}$ VI resonance doublet. As such, they provide a window into the behavior of the WD during an outburst that is not affected by orbitally modulated absorption. AG Draconis is particularly notable for the extreme conversion efficiencies previously derived for the Raman scattering, of order 50\% (Schmid et al. 1999; see, however, Birriel et al. 2000). With our optical spectroscopic monitoring, which began at the peak of the 2006 outburst and continues to the present, we can correlate the behavior of spectral variations and quantify flux variations in the UV from the peak of the outburst through its subsequent decay. Here we concentrate on the most significant new result, the variation of the O VI Raman features during the outburst, how it compared with the helium and Balmer line variations, and what it may reveal about the high energy continuum and activity of the WD.

\section{Observations}

Our optical observational data set consists of spectra taken with the Cassini $1.5 \mathrm{~m}$ at Loiano Observatory with all four grisms (covering 700-8500 , resolution between $0.4 \AA$ to $4 \AA$ per pixel, exposure times from 10 to $1200 \mathrm{~s}$ ), and the $2.0 \mathrm{~m}$ Ondřejov Observatory with the RETICON spectrograph (6400-6950 A, resolution $0.24 \AA$ per pixel, exposure time was typically $1200 \mathrm{~s}$ ). The journal of observations and the tables of measurements are included in the online material. All spectra were reduced using
IRAF and our own special purpose routines written in $\mathrm{IDL}^{1}$. In several instances, contemporaneous spectra allow us to correlate and cross-calibrate the data from the three sites. The continuum variations were checked based on measurements of absorption line equivalent widths from the $\mathrm{K}$ star photosphere and the fluxes were corrected for continuum variations using contemporary CCD photometry.

For phasing we have used the orbital ephemeris of Fekel et al. (2000) based on the K star radial velocities,

$T\left(v_{\text {rad,max }}\right)=2450912.5( \pm 4.1)+548.65( \pm 0.97) E$

with an amplitude of $5.86 \pm 0.30 \mathrm{~km} \mathrm{~s}^{-1}$. This velocity amplitude is resolved only in our TNG spectrum and confirmed by cross correlation of the Ondřejov data as an upper limit $\left(\leq 1.7 \mathrm{~km} \mathrm{~s}^{-1}\right.$ using cross correlations at 6540-6550 ̊).

These new observations were supplemented by archival spectra. An archival HST/STIS medium resolution observation covering much of the UV and optical (1150-8000 $\AA$ with some gaps in spectral coverage) is available (program O6KY, 2003 Apr. 19, MJD 52 748) at orbital phase 0!34. Wavelengths were checked for the $H S T$ /STIS spectra and registered using the interstellar velocities measured for the $\mathrm{Na} \mathrm{I} \mathrm{D} \mathrm{lines} \mathrm{and}$ the published IUE velocities. The FUSE spectra were wavelength corrected using the interstellar $\mathrm{H}_{2}$ absorption lines. We also used a Telescopio Nazionale Galileo (TNG) spectrum, obtained at high resolution on 2005 Aug. 14 (MJD 53 596) at orbital phase 0 p 89 . It nearly coincides with the peak of a minor outburst, with $\Delta U \approx 1.5 \mathrm{mag}$, and $\Delta B \approx 1 \mathrm{mag}$ (Skopal et al. 2007). Continuum points were chosen, in part, based on this spectrum in comparison with the HST/STIS spectrum. It has also been essential for understanding the profiles of the neutral and ionized helium and Balmer lines ${ }^{2}$. Classical photoelectric $U B V R_{\mathrm{C}}$ measurements in the standard Johnson-Cousins system were carried out by single-channel photometers mounted in the Cassegrain foci of 0.6-m reflectors at the Skalnaté Pleso and Stará Lesná (pavilion G2) observatories (see Skopal et al. 2004, for details). The star SAO $16952(V=9.88, B-V=0.56, U-B=-0.04$, $\left.V-R_{\mathrm{C}}=0.32\right)$ and SAO $16935(V=9.46, B-V=1.50$, $U-B=1.89$ ) were used as the comparison and check, respectively. More recently $U B V R_{\mathrm{C}} I_{\mathrm{C}} \mathrm{CCD}$ photometry was obtained with the $0.5-\mathrm{m}$ telescope at the Stará Lesná Observatory (pavilion G1). The SBIG ST10 MXE CCD camera with the chip $2184 \times 1472$ pixels was mounted at the Newtonian focus. The size of the pixel is $6.8 \mu \mathrm{m}$ and the scale $0.56^{\prime \prime} /$ pixel, corresponding to the field of view of a CCD frame about of $24 \times 16$ arcmin. Other details of the CCD photometric reduction were described by Parimucha \& Vaňko (2005). The same comparison stars were used as in Skopal et al. (2007).

\section{Analysis}

The majority of our data set, listed in Tables 1 and 2, consists of low and moderate resolution optical spectra, which include emission lines of the H Balmer series, He I/II, and O VI Raman features. Cross calibration of the data sets is possible since we have overlap during late 2006 between the Loiano and the higher resolution Ondrejov spectra, all of which were during either the

\footnotetext{
1 IRAF is distributed by the National Optical Astronomy Observatories, which are operated by the Association of Universities for Research in Astronomy, Inc., under cooperative agreement with the US National Science Foundation.

2 We will present more details on these, and other, pre-outburst observations in a paper now in preparation.
} 
S. N. Shore et al.: The spectroscopic evolution of the symbiotic star AG Draconis. I.

Table 1a. Journal of Observations. Loiano: He I/II and O VI Raman emission line equivalent widths $(\AA)$.

\begin{tabular}{|c|c|c|c|c|c|}
\hline JD & $\mathrm{He}$ II & $\mathrm{He} \mathrm{I}$ & O VI & O VI & $\mathrm{He} \mathrm{I}$ \\
\hline $2450000+$ & 4686 & 6678 & 6825 & 7080 & 7065 \\
\hline 4159.6 & 20.9 & 3.14 & 6.5 & - & - \\
\hline 4172.7 & 23.4 & 3.38 & 7.9 & - & - \\
\hline 4182.5 & 24.9 & 2.93 & 7.9 & - & - \\
\hline 4202.6 & 24.9 & 3.36 & 8.3 & - & - \\
\hline 4208.6 & 22.4 & 3.14 & 7.6 & - & - \\
\hline 4215.5 & 24.9 & 3.32 & 8.7 & - & - \\
\hline 4235.5 & 28.2 & 3.72 & 10.4 & - & - \\
\hline 4251.5 & 24.9 & 3.81 & 10.4 & - & - \\
\hline 4279.4 & 28.6 & 3.93 & 12.0 & - & - \\
\hline 4293.4 & 28.8 & 4.36 & 11.9 & - & - \\
\hline 4307.3 & 29.8 & 3.93 & 12.0 & - & - \\
\hline 4329.3 & 27.3 & 3.75 & 9.7 & - & - \\
\hline 4357.3 & 24.0 & 3.46 & 7.8 & - & - \\
\hline 4008.3 & $\overline{-}$ & 4.20 & $\leq 0.2$ & - & - \\
\hline 4033.2 & - & 3.81 & 2.4 & - & - \\
\hline 4039.3 & - & 3.38 & 2.7 & - & - \\
\hline 4049.2 & - & 3.54 & 4.2 & - & - \\
\hline 4008.3 & - & 4.18 & $\leq 0.6$ & $\leq 0.2$ & 4.18 \\
\hline 4033.2 & - & 3.50 & 2.0 & 0.7 & 3.69 \\
\hline 4039.3 & - & 3.15 & 2.8 & 0.8 & 3.25 \\
\hline 4049.2 & - & 3.34 & 4.3 & 1.6 & 3.49 \\
\hline 4159.6 & - & 2.93 & 6.3 & 3.3 & 3.29 \\
\hline 4172.7 & - & 3.22 & 7.8 & 3.5 & 3.47 \\
\hline 4182.5 & - & 3.23 & 8.5 & 4.2 & 3.45 \\
\hline 4202.6 & - & 3.53 & 8.1 & 3.9 & 3.36 \\
\hline 4208.6 & - & 3.13 & 7.86 & 3.6 & 3.53 \\
\hline 4215.5 & - & 3.16 & 8.8 & 4.0 & 3.48 \\
\hline 4235.5 & - & 3.78 & 10.4 & 4.5 & 3.89 \\
\hline 4251.5 & - & 3.81 & 10.9 & 4.5 & 4.06 \\
\hline 4279.4 & - & 4.05 & 12.1 & 4.7 & 4.57 \\
\hline 4293.4 & - & 4.15 & 12.0 & 4.7 & 4.53 \\
\hline 4307.4 & - & 4.05 & 13.0 & 4.5 & 4.48 \\
\hline 4329.3 & - & 3.81 & 10.4 & 4.0 & 4.08 \\
\hline 4357.3 & - & 3.51 & 8.2 & 3.7 & 3.28 \\
\hline
\end{tabular}

decline or inter-outburst phases, and of all three sites in early 2007. These permit a test of the effect of reduced spectral resolution on the measurements of the narrow lines, particularly those of He I. We find that the Loiano data systematically overestimate the equivalent width by about $20 \%$ and, in creating the full time sequence, we include this correction in the plots. There is essentially no effect for the broader lines. The majority of the Loiano $\mathrm{H} \alpha$ profiles are saturated, but several obtained near optical maximum and during the first stages of the decline, taken with $10 \mathrm{~s}$ exposure times, yielded reliable measurements and are included in Table 1b. Although the Ondřejov spectra missed the peak of the 2006 event, they provide sufficient coverage of the subsequent evolution. The equivalent width uncertainty was never above $10 \%$, being largest for the O VI $7080 \AA$ feature and $5-10 \%$ for all He I measurements.

The previously mentioned archival HST/STIS medium resolution observation was absolutely calibrated. The fluxes and equivalent widths were measured for comparison with our data (units of: $F(\mathrm{H} \alpha)=7.51 \times 10^{-11} \mathrm{erg} \mathrm{s}^{-1} \mathrm{~cm}^{-2}(E W=111.6 \AA)$; $F($ He I 6678$)=1.27 \times 10^{-12}(E W=-2.08 \AA), F(\operatorname{Raman} 6825)=$ $4.41 \times 10^{-12}(E W=7.29 \AA) ; F($ He I 7065$)=1.64 \times 10^{-12}$ $(E W=2.45 \AA) ; F(\operatorname{Raman} 7080)=2.14 \times 10^{-12}(E W=3.44 \AA)$. The Raman $7080 \AA / 6825 \AA$ line ratio was 0.47 , higher than our outburst values but consistent with other observations taken during quiescence. The $6825 \AA$ line profile was similar to most
Table 1b. Journal of Observations. Loiano: Balmer emission line equivalent widths $(\AA)$.

\begin{tabular}{ccccc}
\hline \hline JD & $\mathrm{H} \alpha$ & $\mathrm{H} \beta$ & $\mathrm{H} \gamma$ & $\mathrm{H} \delta$ \\
\hline 4008.3 & 102.8 & - & - & - \\
4033.2 & 102.4 & - & - & - \\
4039.3 & 113.7 & - & - & - \\
4049.2 & 111.5 & - & - & - \\
4159.6 & - & 21.69 & 10.82 & 5.70 \\
4172.7 & - & 26.00 & 14.57 & 7.80 \\
4182.5 & - & 27.79 & 16.21 & 10.01 \\
4202.6 & - & 27.79 & 16.71 & 10.43 \\
4208.6 & - & 26.88 & 14.93 & 8.03 \\
4215.5 & - & 29.25 & 17.32 & 8.55 \\
4235.5 & - & 35.61 & 20.42 & 11.82 \\
4251.5 & - & 38.14 & 23.47 & 13.97 \\
4279.4 & - & 44.11 & 27.03 & 16.04 \\
4293.4 & - & 44.24 & 27.65 & 14.75 \\
4307.3 & - & 46.12 & 28.70 & 16.48 \\
4329.3 & - & 38.16 & 23.51 & 14.08 \\
4357.3 & - & 29.88 & 16.78 & 8.98 \\
\hline 4172.7 & - & 25.93 & 13.42 & 7.23 \\
4182.5 & - & 28.05 & 16.34 & 8.04 \\
4208.6 & - & 26.47 & 14.90 & 8.71 \\
4215.5 & - & 28.87 & 16.21 & 8.23 \\
4235.5 & - & 34.66 & 21.09 & 10.84 \\
4251.5 & - & 38.13 & 22.91 & 12.48 \\
4279.4 & - & 44.96 & 25.41 & 14.11 \\
4293.4 & - & 43.84 & 25.21 & 13.79 \\
4307.4 & - & 43.19 & 27.27 & 13.69 \\
4329.3 & - & 37.83 & 22.24 & 12.15 \\
4357.3 & - & 28.77 & 16.04 & 8.13 \\
\hline 4293.4 & - & 43.62 & 27.48 & - \\
\hline & & & &
\end{tabular}

quiescent spectra, with an intermediate equivalent width (see below).

\subsection{O VI Raman features}

As mentioned in the introduction, AG Dra has one of the strongest O VI Raman pairs of any known symbiotic system. The variations of these lines are discussed at length in, e.g. L04, G08. Although the disappearance of the features in symbiotics has been noted sporadically in the literature (e.g. Tomov et al. 2000; Burmeister \& Leedjärev 2007, and references therein), to our knowledge this is the first time such an event has been observed in AG Dra or any other system during an outburst that has been followed with sufficient coverage to provide details of the phenomenon. The minimum line strength coincided with the optical peak of the outburst, although we have no spectra prior to optical $\left(U B V R_{\mathrm{C}}\right)$ maximum. We show in Fig. 1 the first Loiano spectrum, on MJD 54 007, and one taken near the end of the decline from the major outburst, MJD 54049 . The Raman $6825 \AA$ feature was absent at the peak of the outburst although the He I $6678 \AA$ and $7065 \AA$ lines have about the same strength. In Fig. 2 we show the variation of the equivalent width for the two Raman features compared with the $U$ and $V$ light curves. In Fig. 3, we show the comparison of our equivalent width measurements with the published results of L04. The $6825 \AA$ feature varied in equivalent width by almost two orders of magnitude while the continuum varied by about a factor of 5; this is, therefore, not a masking effect of the continuum but a real, systematic variation of the line flux during outburst.

The variation of the Raman $6825 / 7080$ ratio, based on the equivalent widths, is shown in Fig. 4. The change is statistically significant and systematic, the first maximum corresponding to 

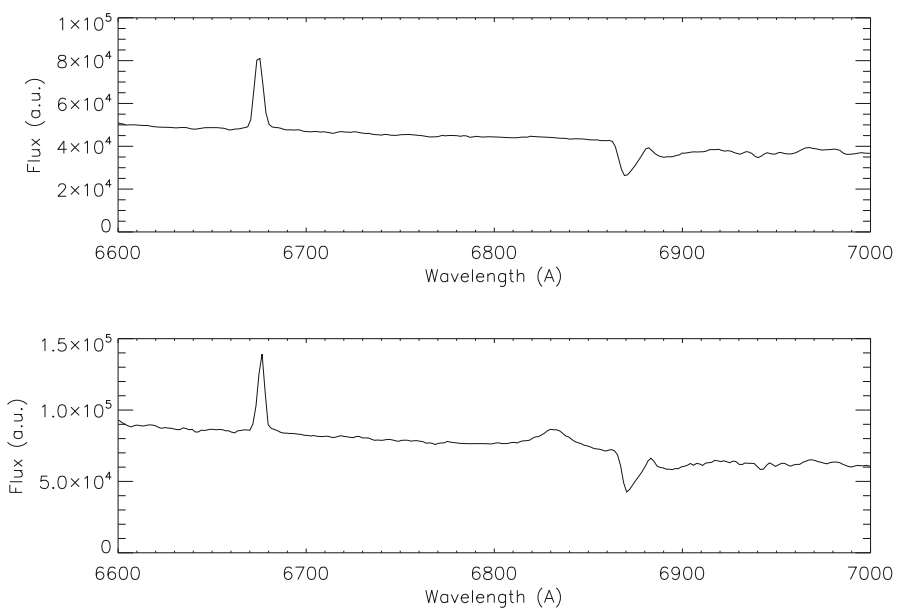

Fig. 1. Comparison of Loiano 4 Å resolution spectra from 2006 Sep. 9 (top) and 2006 Nov. 9 (bottom). The first taken at the peak of the outburst, shows the He I $6678 \AA$ line about as strong as the second spectrum - taken in the decline phase - but with no detectable O VI Raman $6825 \AA$ feature (see text for discussion).
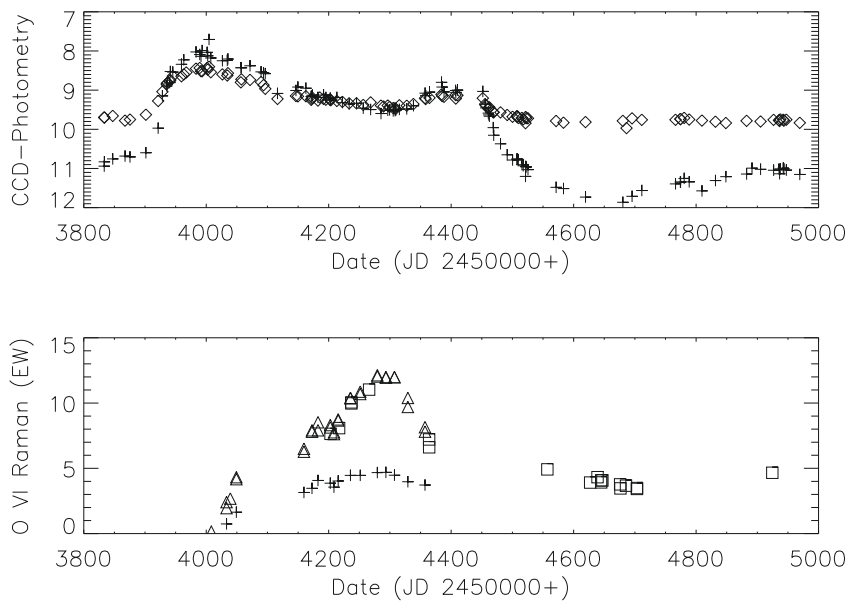

Fig. 2. (Top) $U$ (plus) and $V$ (diamond) CCD photometry for AG Dra throughout the observing interval; (bottom) equivalent width $(\AA)$ variations of the O VI Raman features: $\lambda 6825$ (triangle, Loiano; square, Ondřejov) and $\lambda 7080$ (plus, Loiano).

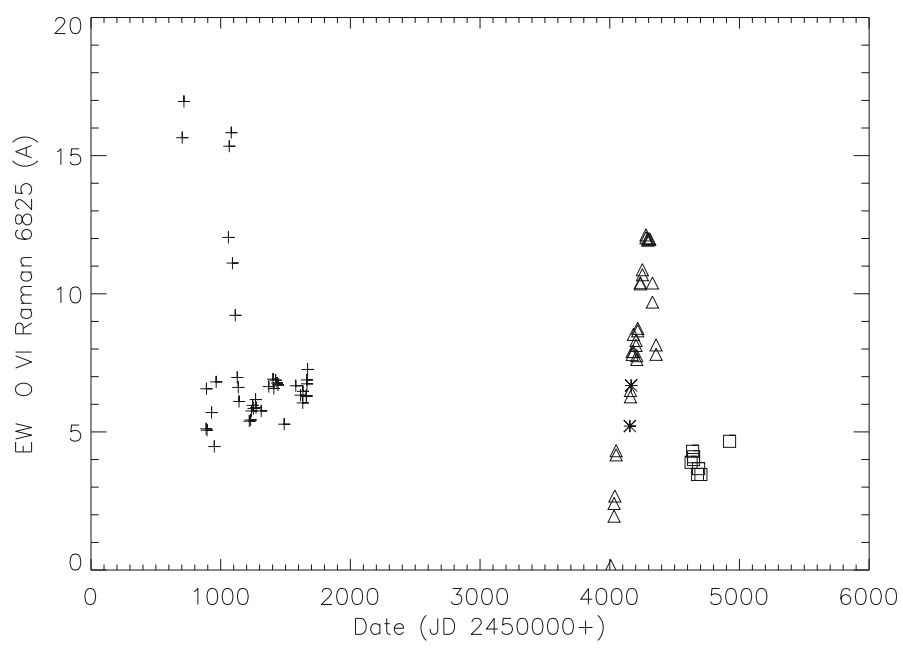

Fig. 3. Comparison of the full data set of blue O VI Raman $\lambda 6825 \AA$ feature equivalent width variations from the present outburst (triangle) and those reported in L04 (plus). The $\mathrm{H} \alpha$ interval was narrower for this comparison.

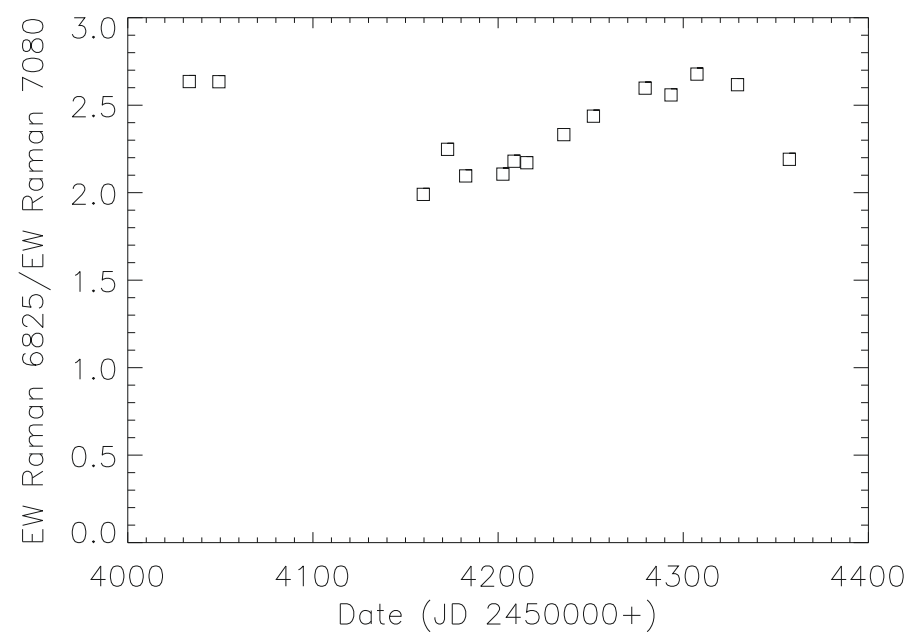

Fig. 4. Variations of the O VI Raman $\lambda 6825 \lambda 7080$ features from the Loiano data set. The minimum value is nearly the same as the $H S T / S T I S$ spectrum obtained during quiescence (see text).

Table 2. Journal of Observations. Ondřejov: emission line equivalent widths (§).

\begin{tabular}{cccc}
\hline \hline JD & H $\alpha$ & He I & O VI \\
$2450000+$ & Wide & 6678 & 6825 \\
\hline 3145 & 76.1 & 1.26 & - \\
3153 & 72.6 & 1.39 & 5.6 \\
3454 & 77.7 & 1.06 & - \\
4203 & 100.4 & 2.62 & 7.6 \\
4217 & 106.4 & 2.51 & 8.1 \\
4237 & 115.5 & 2.98 & 10.1 \\
4266 & 136.3 & 3.34 & 11.0 \\
4364 & 109.7 & 2.57 & 7.2 \\
4557 & 124.1 & 0.88 & 4.9 \\
4595 & 91.4 & 0.96 & - \\
4603 & 90.9 & 0.92 & - \\
4614 & 83.6 & 1.11 & - \\
4623 & 73.1 & 0.97 & - \\
4627 & 78.0 & 1.02 & 3.9 \\
4639 & 72.1 & 0.90 & 4.3 \\
4645 & 76.7 & 1.02 & 4.0 \\
4647 & 66.8 & 1.04 & 4.1 \\
4648 & 75.9 & 1.00 & - \\
4676 & 61.9 & 1.01 & 3.5 \\
4685 & 50.9 & 1.03 & 3.7 \\
4703 & 45.6 & 0.81 & 3.5 \\
4718 & 41.1 & 0.85 & - \\
4761 & 41.1 & 1.04 & - \\
4924 & 65.9 & 1.80 & 4.7 \\
5051 & 47.6 & 0.96 & - \\
\hline & & &
\end{tabular}

the maximum source brightness in our observing interval. Since the last FUSE spectrum from 2007 Mar. 15 nearly coincided with the absolutely calibrated Loiano spectrum of 2007 Feb. 27 we can make some further quantitative statements. The flux ratio of the O VI $\lambda \lambda 1031,1037$ lines in 2007 was approximately the same as the optical Raman features, $\approx 0.45$, which is considerably lower than the peak value that was also characteristic of the earlier FUSE data. Note that although the Raman line varied monotonically with visual magnitude during the 2006 outburst, this trend has not continued, especially during the weaker outburst in 2007 and only now is the emission returning toward its historical quiescent level. Our most recent Ondřejov data, from spring 2009, show that the $\lambda 6825 \AA$ feature has remained 
S. N. Shore et al.: The spectroscopic evolution of the symbiotic star AG Draconis. I.
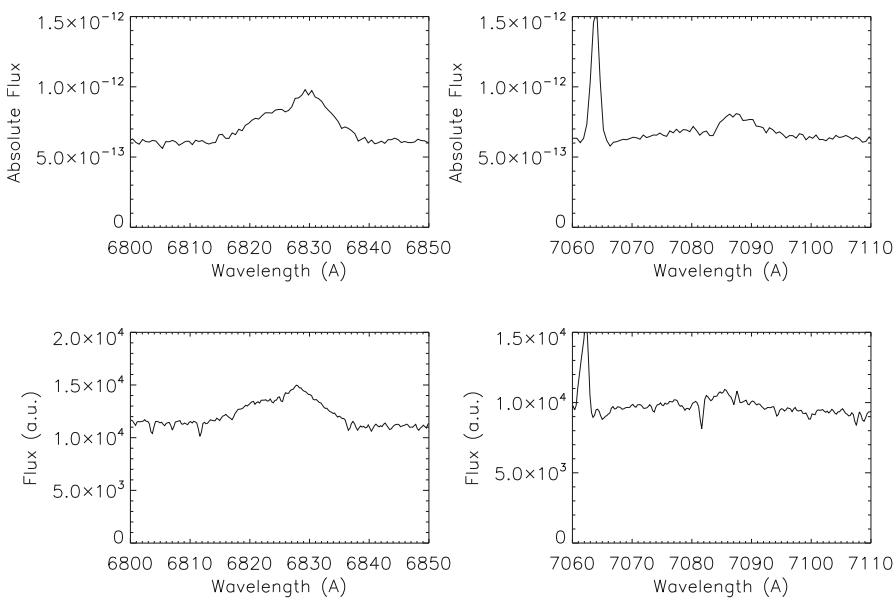

Fig. 5. Comparison of O VI Raman line profiles for HST/STIS spectrum (MJD 52 755) (top) and Ondřjov (MJD 54 704) (bottom). The two spectra were obtained during similar quiescent epochs of AG Dra. The strong emission line blueward of O VI $7080 \AA$ is He I $7065 \AA$.
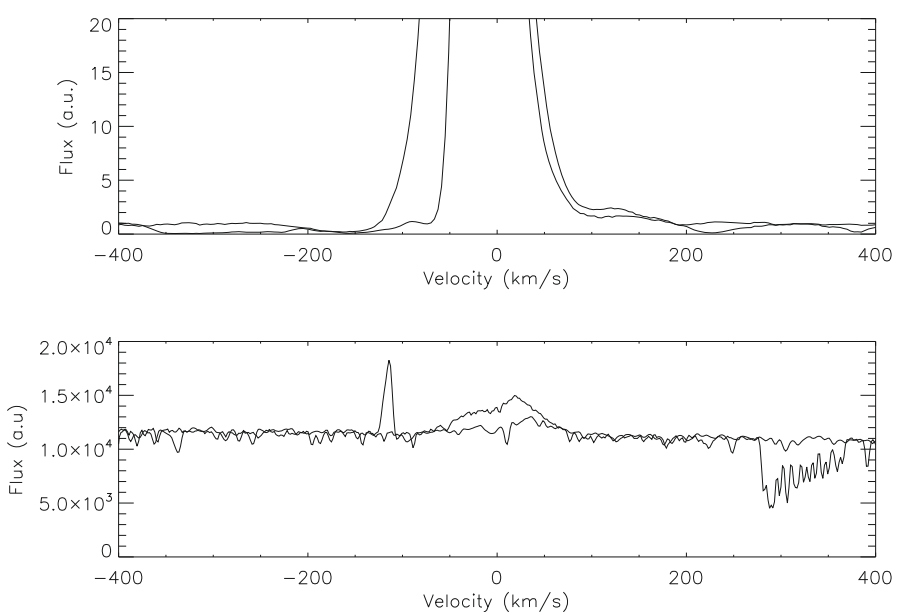

Fig. 6. Comparison of the FUSE O VI doublet spectra (D0090106, 2007 Mar. 15) (top panel: $1031.9 \AA$ solid, $1037.6 \AA$ dot-dash) with the Ondřejov spectrum shown in Fig. 5 (bottom panel: $6825 \AA$ solid, $7080 \AA$ dot-dash). Fluxes are normalized, and the radial velocities for the optical lines have been scaled by the Raman frequency shift (see text for discussion). The narrow emission feature in the bottom panel is He I $7065 \AA$.

below the minimum equivalent width reported in the time series in L04 while maintaining essentially the same profile as Fig. 5. One additional measurement is available for the ratio for MJD 54703 from the Ondřejov spectra, $E W(\mathrm{O}$ VI Raman 7080) = $0.94 \AA$. This corresponds to a $6825 / 7080$ ratio of $\approx 3.7$, the highest value we observed.

An interesting point that emerges from the HST/STIS observation is the separation of the peak of the emission and the "notch" in the two features. The velocity difference, $\left|\Delta v_{\text {rad }}\right|=$ $220 \pm 7 \mathrm{~km} \mathrm{~s}^{-1}$, was quite similar despite the different profiles. This is compatible with from the Ondřejov spectrum obtained during our interval when for the $6825 \AA$ feature the separation was $\approx 200 \mathrm{~km} \mathrm{~s}^{-1}$. We discuss possible origins of this structure in the last section. Figure 6 compares the line profiles for the O VI resonance lines from the last FUS E spectrum with the Ondřejov spectrum of the Raman features (MJD 54 703). The velocities have been corrected for the Raman effect wavelength shift (a factor of 6.6) in the optical spectra.

\subsubsection{Raman conversion efficiency}

We attempted to use the last FUSE data to determine the Raman conversion efficiency that, for AG Dra, has been studied based on data taken in the 1990s. For the Raman features, the measured fluxes from Loiano were $F(6825)=4.45 \times 10^{-12} \mathrm{erg} \mathrm{s}^{-1} \mathrm{~cm}^{-2}$ and $F(7080)=1.94 \times 10^{-12}$. It should be noted that these are almost identical to the values from the $H S T / S T I S$ spectrum, see above. For the FUSE spectrum, the measured fluxes were $F(1031)=1.7 \times 10^{-12} \mathrm{erg} \mathrm{s}^{-1} \mathrm{~cm}^{-2}$ and $F(1037)=0.7 \times 10^{-12}$. Thus, the doublet ratio was similar for the O VI lines, 0.44 for the Raman features and 0.42 for the FUV doublet. The FUSE fluxes are, however, not directly usable for deriving the Raman conversion efficiency, $Y=N(6825) / N(1031)$, based on the comparison in both line and continuum fluxes and the Science Data Assessment form for observation D0090106. If, however, the continuum fluxes scale then it is possible to at least place a limit on $Y$. Using the Hutchings \& Gaisson (2001) and Sofia et al. (2005) FUV extinction curves for the Galaxy, and the mean visual extinction $E(B-V)=0.11$ from Schegel et al. (1998) maps, the extinction correction for AG Dra at the FUV O VI doublet is 6.9. The spectrum was taken around quiescence following the major outburst. If the actual continuum level was approximately the same, we find an additional correction factor of $6 \pm 1$ for the continuum level and $Y \approx 0.5$ and 0.4 for the two components, respectively. If, instead, we adopt $E(B-V)=0.08$ (see Young et al. 2005; Birriel et al. 2000) we find $Y \approx 0.4$ and 0.3 for the two components applying the differential $\mathrm{H}_{2}$ absorption correction factors from Birriel et al. (respectively 0.97 and 0.64 for the two components of the FUV doublet).

This tentative efficiency is similar to that obtained by Schmid et al. (1999), $Y=0.5$ for $\lambda 6825$ and between 0.3 and 0.5 , with an uncertainty of order $50 \%$, from the two observations of $\lambda 7080$ using ORFEUS data obtained in 1993 and 1996; neither was obtained during an outburst. In contrast, this value is larger than found by Birriel et al. (2000), who obtained $Y \approx 0.14$ for the two Raman features using contemporaneous $H U T$ and groundbased spectra from 1995 Mar. 16 when the source was, according to AAVSO data, declining after a sequence of outbursts. The ratio of the O VI FUV doublet lines during the HUT observations, 3.23 , is quite different from ours and about the minimum value obtained during the FUS E era. We do not have coincident FUV data for the peak of the outburst when the O VI Raman feature was undetectable.

\subsubsection{O VI Raman line profiles}

The O VI $\lambda 6825$ line profile appears to consist of two components that vary similarly when the Raman lines are strong. The red component is significantly enhanced relative to the blue, with the ratio of their peaks reaching up to $\approx 2$. As proposed by Schmid et al. (1999), we interpret this as two separate contributors, one from the wind (and possibly accretion disk) of the WD, the other from the ionized region surrounding it within the K star wind. The displacement in the centroid is consistent with the ionized region having a different velocity than the WD wind, the result of a flow through the ionization zone. The centroid of the line is shifted by approximately $+300 \mathrm{~km} \mathrm{~s}^{-1}$, again consistent with scattering from a wind with a velocity of order $50 \mathrm{~km} \mathrm{~s}^{-1}$ relative to the WD. Although the intrinsic Raman profiles are not Gaussian, to parameterize the shape variations we decomposed the Raman $6825 \AA$ using two Gaussians. The $E W$ variations for the two components are shown in Fig. 7. The 


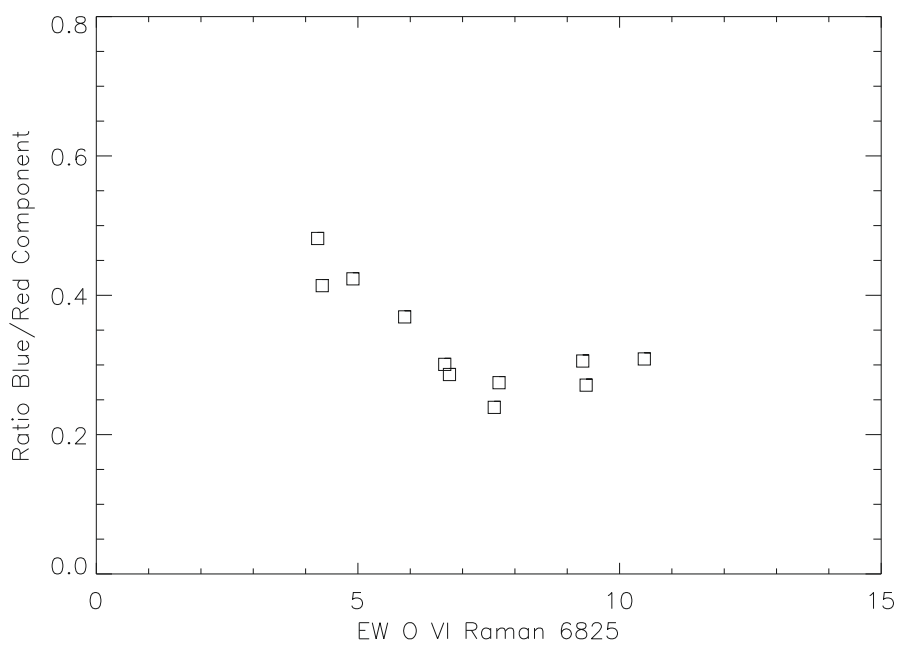

Fig. 7. Ratio of the equivalent widths of the two O VI Raman $6825 \AA$ components obtained by Gaussian decomposition as a function of the line strength (in $\AA$ ). The blue component is both weaker and consistently broader, the broadest profile corresponding to the strongest integrated flux and optical minimum.
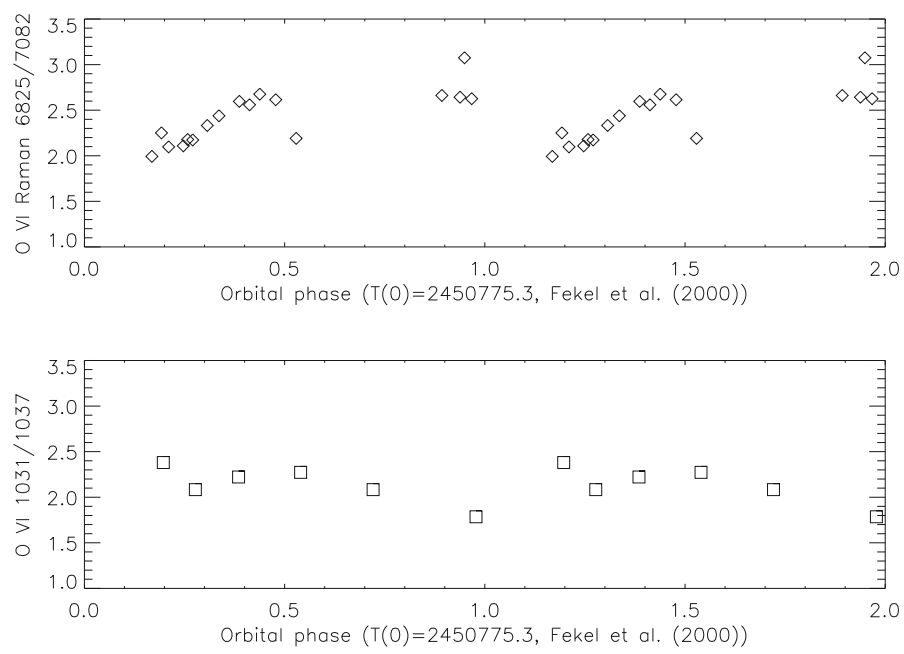

Fig. 8. Flux ratio of the FUV O VI doublet components (bottom) and the optical Raman features (top) as a function of orbital phase based on the Fekel et al. (2000) radial velocity ephemeris; phase 0? 0 being elongation of the K star.

choice of profile was a convenience, in the absence of the FUV doublet we chose this as a way of displaying the relative contributions of the two components. The redward component is always stronger. The minimum value of the ratio in 2004 is about the same as the post-outburst value, 0.27 , while the peak of the ratio occurs at the peak of the minor outburst and at Balmer line minimum. We emphasize, however, that this is a phenomenological characterization of the profile variations, it is also plausible that there is only a single component and the changes reflect, instead, variations in the O VI resonance line profile at $1031 \AA$ due to varying P Cyg absorption on the blueward side of the profile. We do not have sufficient coverage of the $7080 \AA$ Raman feature to compare the variations but, as shown above, the profiles are not the same. The stronger component, at longer wavelengths, is narrower in velocity and more variable than the broad component.

For the interval 1997 to 2003, L04 report a correlation of $\mathrm{H} \beta$ emission equivalent width with orbital period. For comparison,
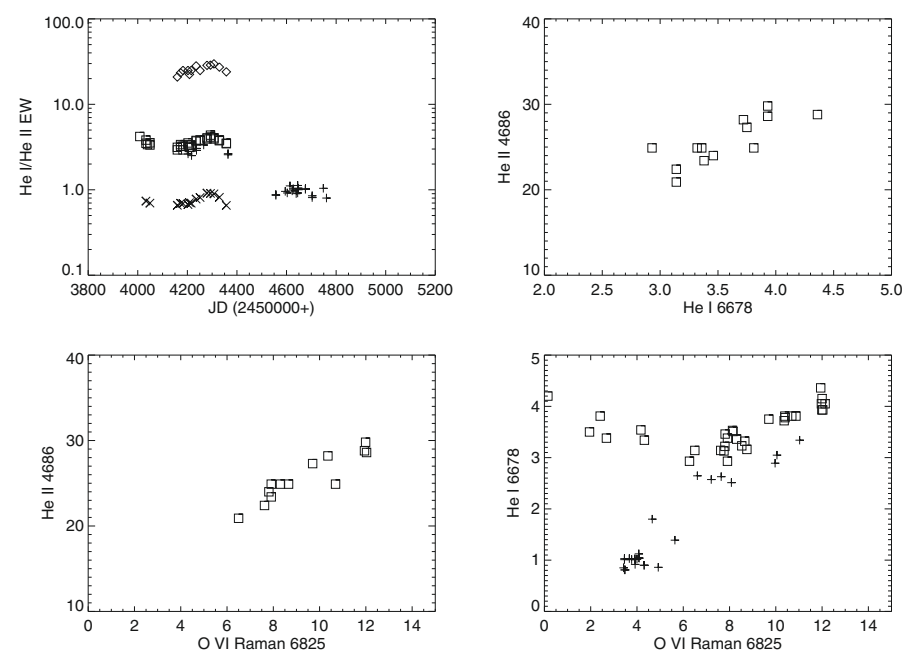

Fig. 9. Equivalent width variations of the He I and He II lines. Upper left: He II 4686 (diamond, Loiano only), He I 6678 (square, Loiano; plus: Ondřejov) and He II 7065 (cross, scaled by 1/5, Loiano only); upper right: He I $6678 \AA$ vs. He II $4686 \AA$ (Loiano); lower left: O VI $6825 \AA$ vs. He II $4686 \AA$ (Loiano only); lower right: O VI $6825 \AA$ vs. He I $6678 \AA$ (square: Loiano, plus, Ondřejov). See text for discussion.

using a different measurement, we show, in Fig. 8, the variation of the O VI FUV doublet and optical Raman line ratios phased on the radial velocity ephemeris (Fekel et al. 2000). The FUV spectra were obtained mainly in quiescence, while our spectra were taken during outbursts so any search for orbital modulation of the line strengths is hindered by the outburst, which lasted about 0 ? 2 . This dominates the relatively brief time interval of these equivalent width measurements and we regard any variations as being due to the outbursts. A dedicated monitoring of this system is needed to assure that a dense enough coverage is available during any quiescent phase to disentangle the effects. If the orbital modulation of the line ratio is real, being a single wave with minimum at $\mathrm{K}$ star elongation may indicate a slight eccentricity in the system or an asymmetry in the WD environment.

\subsection{Helium lines}

Figure 9 shows the variation of the helium lines through the outburst. The He I $7065 \AA$ line showed similar variations but our coverage is not as complete. To highlight the qualitative change in the AG Dra variations, we also show the comparison between the Raman $6825 \AA$ feature and He I $6678 \AA$ (lower right panel). This highlights the difference between the outburst and postoutburst variations, already visible from the time series. The difference is real, the same relative behavior is seen in the $\mathrm{H} \alpha$ variations. This does not appear to be orbital modulation (that would not seriously affect optical lines). Instead, it appears that the excitation significantly changed while the He II $4686 \AA$ line did not show any departure from its earlier behavior. High resolution TNG data, of which we show a sample in Fig. 12 (to be discussed more extensively in a separate paper, in preparation), show absorption on all He I line profiles at about $-50 \mathrm{~km} \mathrm{~s}^{-1}$ (relative to the systematic velocity of $-144 \mathrm{~km} \mathrm{~s}^{-1}$ ) at the same velocity as the Balmer line absorptions. But at most, the variation in the absorption component accounts for $20-30 \%$ of the equivalent width variations. No such feature is seen on the He II $4686 \AA$ profile whose peak corresponds to the peak of the Balmer lines. The He II, at high resolution, is asymmetric with an extended 
red wing, perhaps indicating a weak absorption trough on the approaching side.

The strongest He I lines $(\lambda \lambda 5875,6678$, and $7065 \AA)$ all show a peak coinciding with the He II and Raman features, at the minimum of the visual flux. As reported by L04 and G99, we find $\langle E W(\mathrm{He}$ II 4686$) / E W(\mathrm{H} \beta)\rangle=0.80 \pm 0.17$; at no epoch did this exceed 1.0, but we emphasize that these spectra did not coincide with the peak of the major event. Notably, none of our spectra displayed a He II $4686 \AA$ line as strong as the most extreme reported by L04. The line strength during the declining phase of the outburst was strongly correlated with that of the Raman $6825 \AA$ feature but at line minimum light appears to vary relatively less. There is, in addition, a single late measurement, MJD 54 703, of He I $7065 \AA$ from Ondřejov, $E W($ He I 7065) = $0.89 \AA$, which is the weakest recorded during this observing interval. Birriel (2004) reports the detection of Raman scattered $\mathrm{He}$ II in the spectra of HM Sge and V1016 Cyg. We find no evidence for this feature in any AG Dra spectra, neither the lower resolution Loiano data taken during the outburst nor the high resolution TNG spectra taken outside of outburst.

The He I lines vary in phase with the He II $\lambda 4686$ line during the decay phase of the outburst; we have no simultaneous spectra at peak but there is an additional, weak line is present at $6680.2 \AA$ which coincides with the NIST listing for O IV $6682 \AA$ (at the systemic velocity) but it is more likely He II $6682 \AA$. This line varies but we do not report those results since the $\mathrm{S} / \mathrm{N}$ ratio is not sufficiently high in most of the spectra to provide accurate measurements. Its properties are the same as the helium lines, and we thus identify this as a recombination line from the same ionized region. It appears to be constant in the Ondřejov spectra, where it is barely detectable; because of the low resolution and SNR of the HST/STIS spectrum it is not visible. This line is visible in the velocity plot for He I $6678 \AA$ (Fig. 12).

\subsection{Balmer lines}

All quoted equivalent widths were measured between $\pm 2000 \mathrm{~km} \mathrm{~s}^{-1}$ for consistency with previous reports of extended wings and based, in part, on continuum points selected with the TNG and HST/STIS spectra. The extended wings (from 1000 to $2000 \mathrm{~km} \mathrm{~s}^{-1}$ ) contribute $\approx 25 \%$ of the total flux. Figure 10 shows the variations of the equivalent widths; our coverage of the major outburst only includes $\mathrm{H} \alpha$. Our coverage of the post-outburst period shows that $\mathrm{H} \alpha$ remained below its level during the outburst and decline phase. The minimum value we find is consistent with that reported in L04 (their Fig. 4 shows the variations phased according to the photometric ephemeris). Their equivalent widths for both $\mathrm{H} \alpha$ and $\mathrm{H} \beta$ are greater than any we observed during the recovery from the major outburst. The effects on the interpretation of the $\mathrm{He}$ line variations due to the continuum variations are, however, important and we will return to this point presently.

\subsection{High resolution Balmer and He I line profiles}

The TNG spectrum, with a resolution of $\approx 0.1 \AA$, is capable of resolving the structure on the neutral helium emission lines; none of the Ondřejov or Loiano spectra have sufficient resolution to detect this, nor were they resolved in the medium dispersion HST/STIS spectra. The He I lines are all well resolved and, more significantly, it display complex profiles with an extended red wing and $F W Z I=150 \mathrm{~km} \mathrm{~s}^{-1}$; The absorption
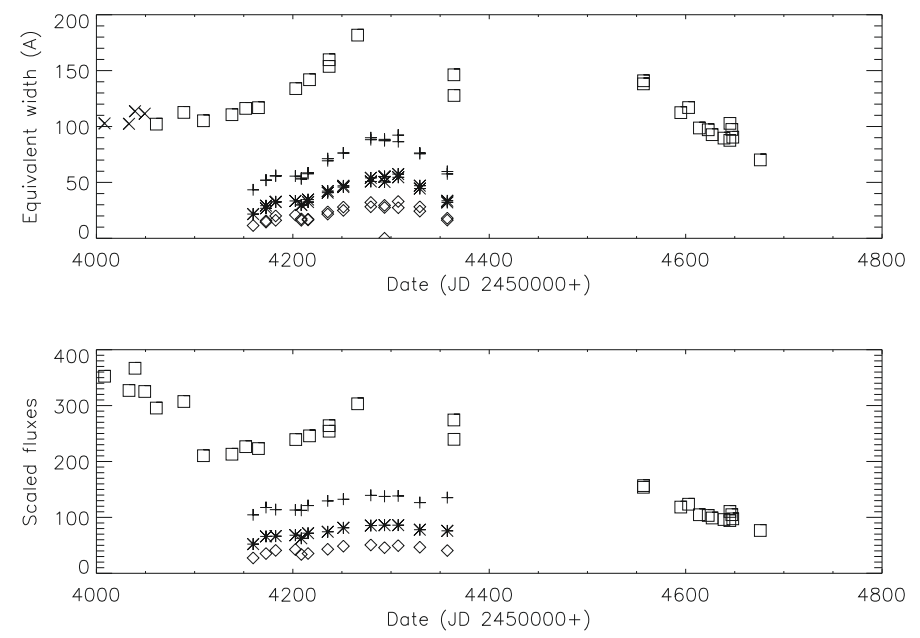

Fig. 10. Variation of the Balmer lines from the beginning of the 2006 outburst of AG Dra; $\mathrm{H} \alpha$ square: Ondřejov, cross: Loiano. All other Balmer line measurements are only from Loiano spectra $-\mathrm{H} \beta$, plus; $\mathrm{H} \gamma$, asterisk; $\mathrm{H} \delta$, diamond. Top panel: equivalent widths $(\AA)$, bottom panel: scaled fluxes (see Sect. 3.5, below).

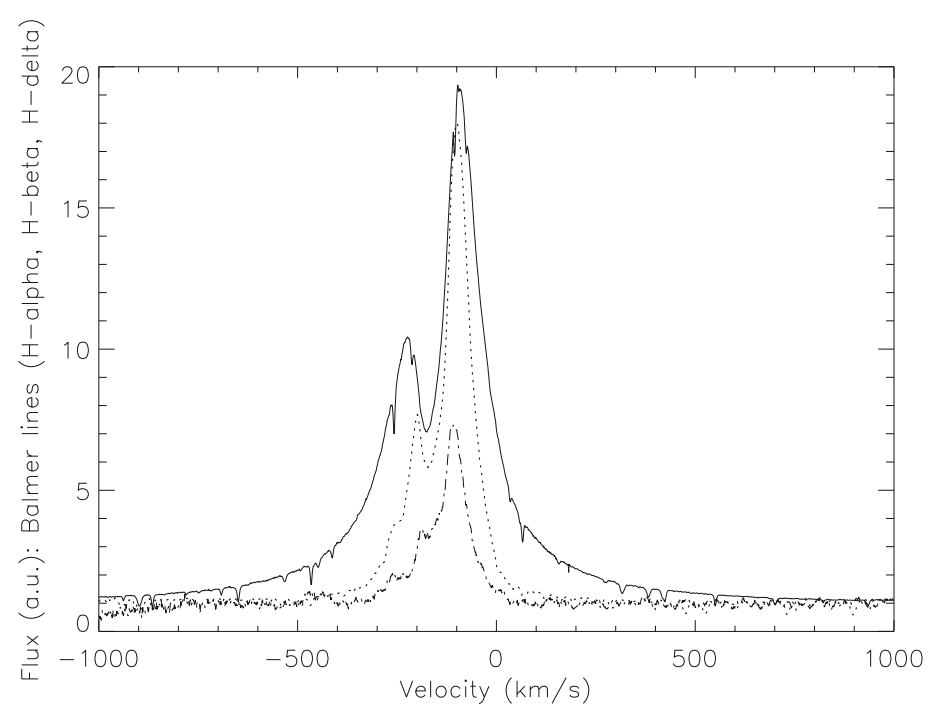

Fig. 11. Sample Balmer line profiles, TNG spectra, 2005 Aug. 14 (MJD 3596). Solid: $\mathrm{H} \alpha$, dotted: $\mathrm{H} \beta$, dashed: $\mathrm{H} \delta$.

feature is at virtually the same radial velocity for the three lines, $-158 \pm 3 \mathrm{~km} \mathrm{~s}^{-1}$, while the peak velocity is $-125 \mathrm{~km} \mathrm{~s}^{-1}$ for He I $5875,-143 \mathrm{~km} \mathrm{~s}^{-1}$ for He I 6678, and $-130 \mathrm{~km} \mathrm{~s}^{-1}$ for He I 7065. The $\mathrm{H} \alpha$ line shows strong absorption at $-177 \mathrm{~km}^{-1}$, but with the peak velocity displaced to $-92 \mathrm{~km} \mathrm{~s}^{-1}$, about $50 \mathrm{~km} \mathrm{~s}^{-1}$ to the red of the systemic velocity. Absorption is present on all He I lines regardless of multiplicity; as shown in Fig. 12, the absorption decreases in order of He I $\lambda \lambda 5875,7065$, and 6678. All He I lines are extremely narrow compared to the Balmer profiles, $F W H M(\mathrm{He} \mathrm{I}) \approx 80 \mathrm{~km} \mathrm{~s}^{-1}$. In contrast, the He II $4686 \AA$ profile is single peaked at the system velocity with a $F W H M=70 \mathrm{~km} \mathrm{~s}^{-1}$ in the TNG and the HST/STIS, spectra, although taken at activity states of the AG Dra. These are consistent with the lines being formed near the WD but with a puzzle regarding the cause of the absorption. It cannot be from the wind of the red giant and must, instead, arise within the embedded ionized region around the WD In the Ondřejov spectra, the $\mathrm{H} \alpha$ profiles display variable absorption (e.g. Smith \& Bopp 1981; 

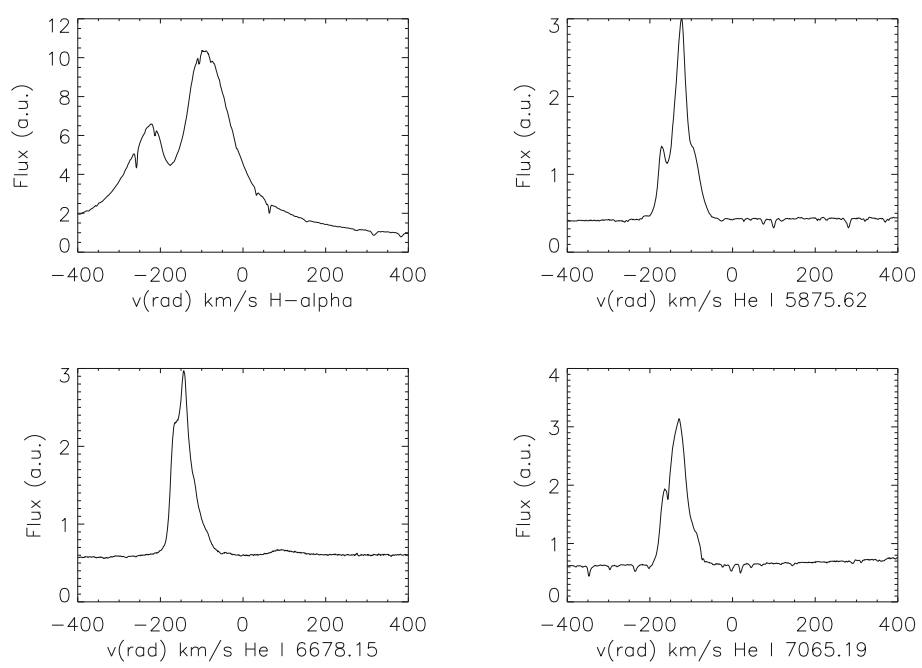

Fig. 12. Comparative line profiles for $\mathrm{H} \alpha$ and the red $\mathrm{He} \mathrm{I}$ lines in the TNG spectra, 2005 Aug. 14 (MJD 3596). The weak feature to the red of He I 6678, at $v_{\text {rad }} \approx+110 \mathrm{~km} \mathrm{~s}^{-1}$, is identified as He II 6682. See text for discussion.

Ivison et al. 1994; and Belczynski et al. 2000). We reserve further analysis of these variations for the next paper.

\subsection{Line flux variations based on broadband photometry}

With the exception of the major outburst, line variations can be appropriately characterized by equivalent widths alone. However, for the outburst interval, from MJD 4000 to 4400, the continuum variations alter the interpretation of the line strengths for the Balmer and helium lines. We have adopted a scaling based on calibrated spectra taken outside of outburst along with the two in this sequence, from Loiano at the extremes of the major outburst (Sep. 2006 and Feb. 2007) and in the $B, V$, and $R_{\mathrm{C}}$ photometry. Assuming that the visible continuum and emission lines are independently varying, we correct the continuum level by dividing by the continuum flux (based on the photometry), normalized to the minimum $V$ and $R$ magnitudes (using $V$ and $\Delta R_{\mathrm{C}}$ light curves) around $2007 \mathrm{Feb} .15$, roughly coincident with the last FUSE spectrum and a date for which we have a flux calibrated red spectrum. In Fig. 10 (bottom panel) we show the effect on the Balmer lines, in Fig. 13 for the Raman $6825 \AA$ feature, in Fig. 14 on the He I $6678 \AA$ line, and in Fig. 15 for He II $4686 \AA$. As discussed above, the Loiano spectrum from MJD 54159 was absolutely calibrated. The Balmer series fluxes were, in units of $10^{-12} \mathrm{erg} \mathrm{s}^{-1} \mathrm{~cm}^{-2}, F(\mathrm{H} \alpha)>62$ (saturated), $F(\mathrm{H} \beta)=10.4, F(\mathrm{H} \gamma)=3.67, F(\mathrm{H} \delta)=1.94$. For the helium lines, in the same units, $F(\mathrm{He}$ I 5578) $=2.54, F(\mathrm{He}$ I 6678 $)=$ $1.95, F(\mathrm{He}$ I 7065$)=2.51$, and $F(\mathrm{He}$ II 4686$)=9.04$. All fluxes quoted are uncorrected for the (minimal) visual reddening.

The main effects of the correction are to reduce the interval of the weak Raman emission phase and to change the variations of He II $4686 \AA$ relative to the He I lines. During the maximum of the major outburst, the upper limit for the $E W \mathrm{~s}$ of both Raman lines is more than an order of magnitude lower than the strongest emission (quiescence). During the decline phase the ratio of the two Raman features remains approximately constant. As reported by L04, the He II $4686 \AA$ line is positively correlated with the Raman emission but once the correction is applied for the continuum variations this is not as tight a relation, a result of the correlation induced by the continuum variations.
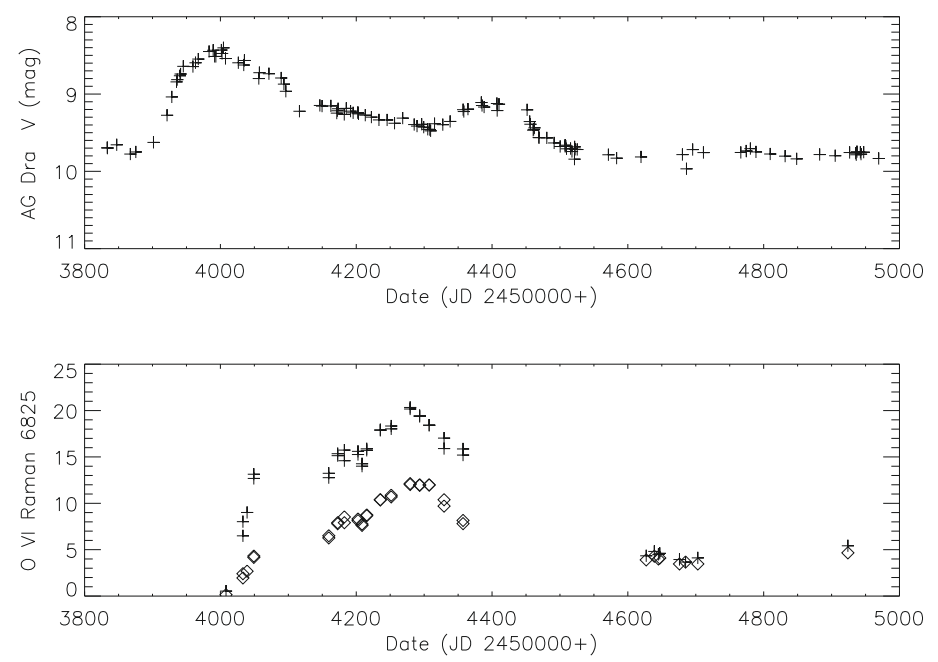

Fig. 13. Top: CCD-V magnitudes for AG Dra during outburst and recovery (see text); bottom: variations of the O VI Raman $6825 \AA$ feature with and without correction to normalized fluxes based on the optical light curve. Diamond: combined Loiano/Ondřejov equivalent widths; plus: scaled fluxes generated based on the $V$ photometry.

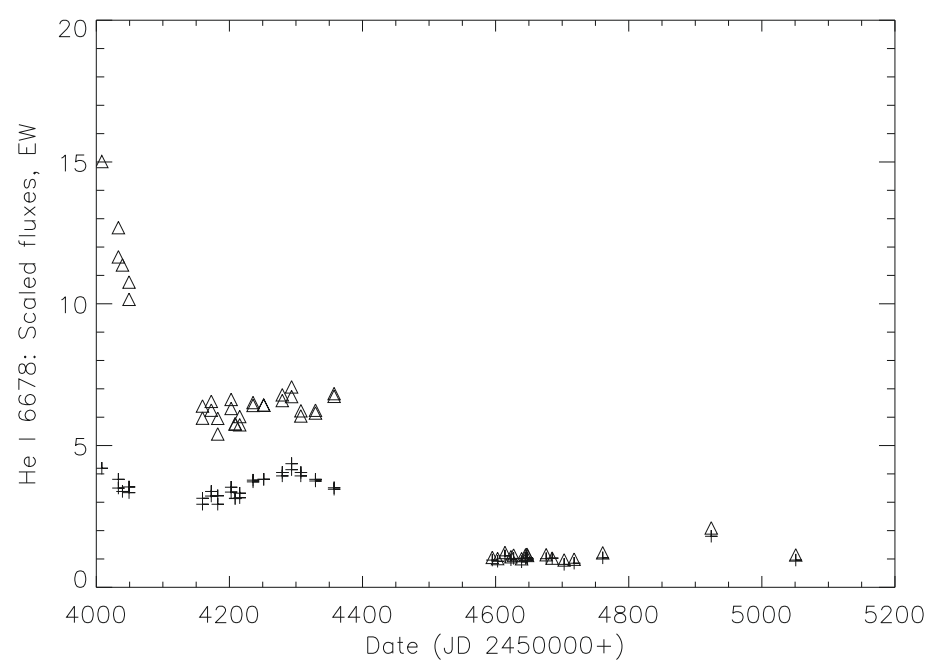

Fig. 14. Comparative variations of the equivalent widths of the He I 6678 line with and without correction to normalized fluxes based on the optical light curve. Triangles, squares: Loiano/Ondřejov scaled fluxes; plus, equivalent widths uncorrected.

The neutral helium lines vary in phase with He II $4686 \AA$ at the strongest phases (Fig. 9). The variations of the Balmer lines are largely unaffected since our Loiano data for $\mathrm{H} \beta-\mathrm{H} \delta$ cover only the decline and quiescence stages of the outburst.

\section{Discussion}

Our results both confirm and extend previous studies of the line variations in AG Dra, especially L04 and G08, but also present important novelties. The most significant is the vanishing - and recovery - of the Raman lines during and following the peak of the major outburst. Since there is no indication that the wind of the red giant has significantly changed in velocity or density, the hydrogen line variations are consistent with the same profile although the line flux decreases, it is more likely that this signals the disappearance of the emission component of the O VI resonance lines. This observation provides further support for the 
S. N. Shore et al.: The spectroscopic evolution of the symbiotic star AG Draconis. I.

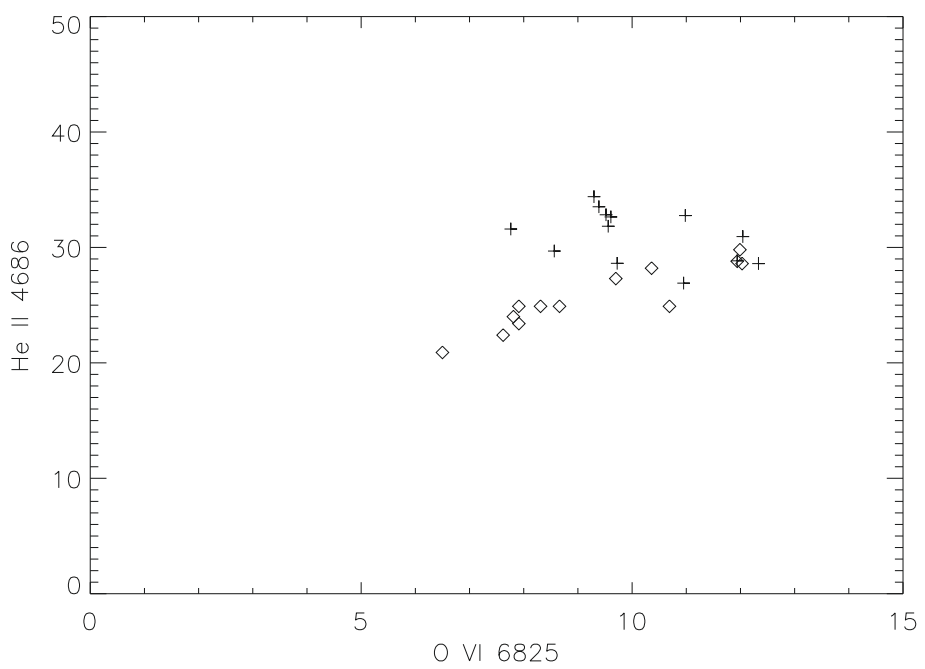

Fig. 15. Comparative variations of the equivalent widths of the O VI Raman $6825 \AA$ line with He II $4686 \AA$ based on the $B$ and $V$ photometric variations. Plus: equivalent widths; diamond: scaled fluxes.

Raman line as a proxy measure of the state of the highest ionization region and inner fast wind around the gainer.

The minimum Raman line equivalent width during the decade before the 2006 outburst was about $6 \AA$, about the same as our first Ondřejov spectrum and the HST/STIS measurement. During the entire recent outburst, we observed consistently lower values. This can not be due to continuum variations alone, although some may be present at about the $20 \%$ level based on the variations of the He I lines. If each of the Raman features consists of two components, as suggested by the decomposition, then the line variations indicate that both the WD wind and ionized region disappeared during the peak of the major outburst. Since we do not have observations prior to the photometric peak we cannot constrain the density from the time dependence alone. The recovery of the emission line was more rapid than the rate of continuum decrease, seen from the comparison of the light curve with the Raman flux variations.

The suggestion that the soft XR and UV variations are due to expansion and falling effective temperature of the gainer in this system receives support from the behavior we observe (Greiner 1997; Gonzalez-Riestra et al. 1999). The WD develops an optically thick, extended atmosphere with a large covering factor that suppresses the resonance $O$ VI lines and the ionized region but maintains sufficient excitation to produce the emission at He I. The conversion efficiency for UV photons by Raman scattering has been obtained from previous observations of the system during quiescence and unless there is a drastic change in the structure of the wind in the accretion region we would expect it to remain roughly constant, so the disappearance of the optical Raman lines indicates an increase in the optical depth of the $\mathrm{UV}$ and the redistribution of the radiation to longer wavelengths. If the redistribution is essentially passive - that is, if during the outburst the bolometric luminosity of the WD does not significantly change - the maximum increase we would expect in the visible is about one magnitude. The brightest outbursts are substantially larger than this, using $U$ and $B$ as the proxies for the redistribution since the red giant contributes most of the flux at $V$ and longer wavelengths. Instead, if a thermonuclear runaway is initiated on the gainer, the increase in the radius of the pseudophotosphere will produce a substantial change in the ultraviolet spectrum, including the appearance of neutral and low ionization state lines of the iron peak (the so-called "iron curtain" well known from classical novae and Luminous Blue Variables) and the disappearance of the highest ionization wind lines that should normally be visible from the gainer. This antiphase behavior, so well known from LBVs, is consistent with IUE and ROS AT observations reported by Greiner et al. (1997) and G08 during the inter-outburst period preceding the latest eruption. To increase the optical depth sufficiently in the UV requires column densities of order $10^{24} \mathrm{~cm}^{-2}$ or higher and would be consistent with an expansion of the WD photosphere accompanied by an optically thick wind.

Lee \& Kang (2007) propose an accretion disk as the source for Raman scattered photons and model the inequality of the peaks as an optical depth effect by self-absorption, in the RG wind. It is possible that this is a contributor to the spectra, hydrodynamical modeling of symbiotic wind does produce disks (e.g. Walder et al. 2008), but it is far more direct to decompose the profile into one from the scattering of the WD wind and the other from the surrounding ionized nebula of the K star wind. the intensity ratio of the presumed two peaks is slightly variable in the Ondřejov spectra, depending on the line strength. The Raman $6825 \AA$ line profile is consistent with the interpretation by Schmid et al. (1999), based on the comparison of ORFEUS and optical spectra, that the source photons arise from the ionized region around the hot component and are scattered in the slow wind of the $\mathrm{K}$ giant. This would imply that the timescales for the variations should be of order the recombination time and that the outburst, extinguishing the FUV and soft XR, causes a collapse of the $\mathrm{O}^{+5}$ region around the WD. Additional changes on a shorter timescale, mainly in the broader component, could be due to the same mechanism that produces the outburst, that the cooling of the pseudo-photosphere and the increase in the fast wind optical depth emission from the Raman lines. The $7080 \AA$ feature has a different profile. We suggest that in addition to the intrinsic WD wind absorption component, and that of the ionized region in the RG wind, there are additional absorbers both in the ionized and neutral K star wind - that are altering the Raman profile. Several multiplets of S I ground state transitions between 1030 and $1038 \AA$ and the C II 1036, $1037 \AA$ doublet may not be entirely interstellar in origin. Instead, any additional absorption would not only alter the Raman line profile but also affect the line ratio. If, for instance, during a major outburst the C II absorption line varies, this will affect the conversion efficiency and the resulting optical profiles. Since, however, the region is heavily blanketed by interstellar $\mathrm{H}_{2}$ it is not possible to obtain an absorption equivalent width and lacking contemporaneous pairs of FUV and optical spectra during the peak of the outburst, this must remain an hypothesis.

The timescale for an outburst, about four months, is approximately the recombination time for a region with an electron density of about $10^{8} \mathrm{~cm}^{-3}$ for the $\mathrm{O}^{+5}$ zone, using the recombination coefficients from Nahar \& Prasad (2003) in the temperature range $15-100 \mathrm{kK}$ and consistent with the previous electron density estimates in the literature, e.g. Young et al. (2005, 2006). This would explain the absence of the [O III] lines in a region sufficiently ionized to otherwise show them. The He II $6682 \AA$ line, weakly present in the best exposed spectra, is slightly stronger relative to He I $6678 \AA$ in data from 2008 June, possibly indicating ionization has occurred. The data from the main outburst do not have sufficient resolution to show this line, and there are no contemporaneous UV spectra during a similar phase (although the one FUSE spectrum, taken almost at quiescence, shows the O VI resonance lines at about their maximum strength). This may explain the weakening of the red component 
of the Raman line. The disappearance of both components of the Raman features also challenges shocked wind models for events during the outburst that must produce temperatures lower than those of the photoionization source. the increase in the visual flux, $0.3-1.0 \mu$, and the contemporary decrease in the ultraviolet are compatible with simple flux redistribution from an optically thick pseudo-photosphere mentioned above. This would easily account for the disappearance of the high ionization features and the persistence of the He II and He I emission during optical maximum. Further discussion of the long term history of all emission lines and modeling of this system is postponed to the next paper.

Note added in revision: A parallel study of the 2006-2008 outburst was published after we had submitted our paper (Munari et al. 2009, PASP, 121, 1070) that reports photometric and low resolution spectroscopic observations of the event. Their spectrum from near the peak of the outburst, 2006 Sep. 30 (MJD 54 008) is simultaneous with ours at the peak of the outburst and confirms the absence of the two O VI Raman features, identifying the event as a "cool outburst" in the sense of G08. Their subsequent spectra confirm the recovery of the line. They also report, based on an echelle spectrum $(R=35000)$ taken on 2006 Oct. 6, the presence of P Cyg components on the He I lines with velocities similar to those we describe here. A further discussion of these results will be included in our next paper.

Acknowledgements. Research at Loiano is supported by INAF. P.K. was supported by ESA PECS grant No. 98058. G.M.W. acknowledges support from NASA grant NNG06GJ29G. We thank the staff at Loiano for their kind help in obtaining many of the spectra used in this study as a service observing program and U. Munari, C. Rossi, and R. Viotti for use of unpublished spectra. Some spectra at Ondřejov were taken by M. Netolický, B. Kučerová, V. Votruba and D. Korčáková. M.W. was supported by the Research Program MSM0021620860 of the Ministry of Education of the Czech Republic. A.S. was supported by a grant of the Slovak Academy of Sciences No. 2/7010/27. We thank T. B. Ake for collaboration on the FUSE proposal that began this observation, unfortunately not executed before the end of the mission, and for his advice on the FUSE data, and J. P. Aufdenberg, C. Rossi, and R. Viotti for valuable discussions.
The HST/STIS and FUSE spectra were obtained from the MAST archive of STScI and archival visual photometric data were provided by the AAVSO.

\section{References}

Belczynski, K., Mikoajewska, J., Munari, U., Ivison, R. J., \& Friedjung, M. 2000, A\&AS, 146, 407

Birriel, J. J. 2004, ApJ, 612, 1136

Birriel, J. J., Espey, B. R., \& Schulte-Ladbeck, R. E. 2000, ApJ, 545, 1020

Burmeister, M., \& Leedjärv, L. 2007, A\&A, 461, L5

Eriksson, M., Johansson, S., \& Wahlgren, G. M. 2006, A\&A, 451, 157

Fekel, C. F., Hinkle, K. H., Joyce, R. R., \& Skrutskie, M. F. 2000, AJ, 120, 3255

Gális, R., Hric, L., \& Petrk, K. 2004, RMxAC, 20, 242

Gonzalez-Riestra, R., Viotti, R., Iijima, T., \& Greiner, J. 1999, A\&A, 347, 478 (G99)

Gonzalez-Riestra, R., Viotti, R. F., Iijima, T., et al. 2008, A\&A, 481, 725 (G08) Greiner, J., Bickert, K., Luthardt, R., et al. 1997, A\&A, 322, 576

Hutchings, J. B., \& Gaisson, J. 2001, PASP, 113, 1205

Ivison R. J., Bode, M. F., \& Meaburn, J. 1994, A\&AS, 103, 201

Lee, H.-W., \& Kang, S. 2007, ApJ, 669, 1156

Leedjärv, L., Burmeister, M., Mikolajewski, M., et al. 2004, A\&A, 415, 273 (L04)

Meinunger, L. 1979, Inf. Bull. Var. Stars, 1611

Mikolajewska, J., Kenyon, S. J., Mikolajewski, M., Garcia, M. R., \& Polidan, R. S. 1995, AJ, 109, 1289

Nahar, S. N., \& Prasad, A. 2003, ApJS, 149, 239

Parimucha, Š., \& Vaňko, M. 2005, CoSka, 35, 35

Robinson, L. 1969, Per. Sviosdi, 16, 507

Schlegel, D. J., Finkbeiner, D. P., \& Davis, M. 1998, ApJ, 500, 525

Schmid, H. M., Krautter, J., Appenzeller, I., et al. 1999, A\&A, 348, 950

Skopal, A., Pribulla, T., Vaňko, M., et al. 2004, CoSka, 34, 45

Skopal, A., Vanko, M., \& Pribulla, T. 2007, AN, 328, 909 (Sk07)

Smith, S. E., \& Bopp, B. W 1981, MNRAS, 195, 733

Smith, V. V., Cunha, K., Jorissen, A., \& Boffin, H. M. J. 1996, A\&A, 315, 179

Sofia, U. J., Wolff, M. J., Rachford, B., et al. 2005, ApJ, 625, 167

Tomov, T., Munari, U., \& Marrese, P. M. 2000, A\&A, 354, L25

Tomov, N. A., \& Tomova, M. T. 2002, A\&A, 388, 202

Viotti, R., Ricciardi, O., Giangrande, A., et al. 1983, A\&A, 119, 285

Viotti, R. F., Friedjung, M., \& González-Riestra, R. 2007, BaltA., 16, 20

Walder, R., Folini, D., \& Shore, S. N. 2008, A\&A, 484, L9

Young, P. R., Dupree, A. K., Espey, B. R., Kenyon, S. J., \& Ake, T. B. 2005, ApJ, 618, 891

Young, P. R., Dupree, A. K., Espey, B. R., \& Kenyon, S. J. 2006, ApJ, 650, 1091 